\title{
Diagnostic Value of Test Tilt in Unexplained Syncope-A Heart Institute Abidjan
}

\author{
Justin Djinguin n'dri Ben Koffi \\ Institut de Cardiologie d'Abidjan, Abidjan, Cote d'Ivoire \\ Email: koffidjinguin@yahoo.fr
}

Received 1 November 2015; accepted 16 November 2015; published 23 November 2015

Copyright (C) 2015 by author and OALib.

This work is licensed under the Creative Commons Attribution International License (CC BY).

http://creativecommons.org/licenses/by/4.0/

(c) (i) Open Access

\section{Abstract}

Objective: To evaluate the diagnostic and prognostic contribution of tilt testing in the assessment of unexplained syncope. Patients and Methods: All patients who had a tilt test at Abidjan Heart Institute between October 2014 and May 2015 (a period of eight months) were included and followed in this study. During the monitoring period, patients were interviewed in consultation and examination room. Results: A tilt test was performed in 60 patients with mean age of $45 \pm 20$ years, with a sex ratio of a man for a woman referred to our service for exploration of unexplained syncope. The tilt in dorsal decubitus test was negative in all patients. In inclined position the test was positive in 10 patients $(17 \%)$, and then in inclined position with instillation of glyceryl trinitrate the test was positive in 35 patients $(58 \%)$. The tilt test was positive in 45 patients or $75 \%$. The responses were observed vaso-depressive response in 22 patients, a mixed response in 8 patients, and cardio-inhibitory response in 15 patients. After three months follow-up, through lifestyle changes, through recognition of early symptoms, regular rehydration by the eviction of confined atmospheres, and the reduction of antihypertensive treatment, adoption of the dorsal decubitus or position sitting at the onset of symptoms, $95 \%$ of patients had not presented any recurrence of symptoms. The incidence of recurrence was the same regardless of the type of response to the tilt test. Conclusion: The tilt test is easily achievable but disregarded under review and used in our health centers. It has some diagnostic value in exploring the unexplained syncope. The syncope recurrence rate after a tilt test is very low.

\section{Keywords}

Tilt Test, Unexplained Syncope

Subject Areas: Cardiology 


\section{Introduction}

Le test d'inclinaison est une procédure diagnostique couramment utilisée dans la recherche d'une syncope vaso-vagale. C'est un examen validé dans le diagnostic des syncopes inexpliquées et comporte trois périodes: une période de décubitus dorsal de 14 minutes suivie d'une verticalisation avec une inclinaison à $60^{\circ}$ pendant 30 minutes. En l'absence de syncope après 20 minutes de verticalisation, $0.3 \mathrm{mg}$ de nitroglycérine sublinguale est administrée pour provocation médicamentause pendant 10 minutes.

Malgré sa forte rentabilité, il demeure encore largement sous-utilisé [1] [2].

La méconnaissance de cet examen dans nos centres de santé en Côte d’Ivoire et la durée de l'examen sont en partie les causes dans cette sous-utilisation.

Un "rôle thérapeutique” du test d’inclinaison a été évoqué par certains auteurs devant la diminution significative de l'incidence des épisodes syncopaux lors du suivi des patients [3]. Un apport pronostique du test d’inclinaison a été également suggéré.

Une réponse positive au test d'inclinaison serait un indicateur de mauvais pronostic pour les récurrences de syncope [4].

Les objectifs de notre étude étaient d'évaluer l'apport diagnostique du test d'inclinaison à l'Institut de cardiologie d’Abidjan, et d'apprécier l'évolution des récidives de syncopes notamment en termes de nombre de récidive.

\section{Patients et Méthode}

Il s’agit d'une étude prospective réalisée à l'Institut de cardiologie d'Abidjan au service des explorations fonctionnelles cardiologiques non invasives. Les patients inclus dans cette étude étaient ceux adressés dans le centre pour réalisation d'un test d'inclinaison et les patients hospitalisés pour une affection et présentant des syncopes inexpliquées. Tous les patients adressés étaient mis en observation dans le service de Médecine de cardiologie avant la réalisation du test et bénéficiaient d'un bilan avant ou pendant la mise en observation de 24 heures. Ce bilan qui comprenait une anamnèse, un examen clinique, un électrocardiogramme (ECG) 12 dérivations, une holter ECG de 24 heures, et une échocardiographie n’avait pas retrouvé d’étiologie. Le test d’inclinaison a été réalisé chez tous les patients en respectant les trois étapes. Chaque patient a eu une période de décubitus dorsal de 14 minutes suivie d'une verticalisation avec une inclinaison à $60^{\circ}$ pendant 30 minutes. En l'absence de syncope après 20 minutes de verticalisation, $0.3 \mathrm{mg}$ de nitroglycérine sublinguale est administrée pour provocation médicamenteuse. L’élément diagnostique le plus important du test d’inclinaison est la reproduction des symptômes habituels. Ces symptômes, pour être rattachés à une origine vasovagale, doivent s’accompagner de modifications objectives: chute de la pression artérielle systolique d'au moins $50 \mathrm{mmHg}$ avec apparition de symptômes, expliquant la réponse vaso-dépressive. Parfois une chute de $30 \mathrm{mmHg}$ associée à une bradycardie expliquant la réponse mixte, ou on assistera à une bradycardie sévère avec pause à l'électrocardio- gramme chez patient en syncope nécessitant des mesures urgentes de réanimation. L'absence de modifications objectives en présence des symptômes cliniques habituels, en général des lipothymies, n’est pas sans intérêt car elle oriente vers une cause psychologique (“pseudo syncopes” psychogène) [5]. Tous les patients avaient bénéficié d’un suivi en consultation. Les critères de jugement étaient le diagnostic de malaises vaso-vagaux ou de syncope vasovagale, la présence ou pas de récidive de malaise ou de syncope selon les résultats du test d’inclinaison, et l’évaluation du nombre de récidives selon ces résultats.

\section{Résultats}

\subsection{Caractéristiques Epidémiologiques}

L’âge moyen des patients était de $45 \pm 20$ ans. Notre population d'étude était dominée par le genre féminin (31 femmes pour 29 hommes) avec un sex ratio de 0.93 .

\subsection{Résultats du Test D'Inclinaison}

\subsubsection{Avant le Test D’Inclinaison}

Le nombre moyen de syncopes par mois par personne, avant la réalisation du test d'inclinaison était de $3 \pm 2$. Les symptômes de ces patients étaient en moyenne depuis $6 \pm 3$ mois. Dix neuf patients (31.7\%) présentaient une hypertension dont cinq recevaient au moins un diurétique. Les patients inclus n’avaient pas de cardiopathie 
identifiable. La fraction d'éjection moyenne était de 60\% ¥ 3\%. L’ECG était jugé normal chez 55 patients (92\%) des patients (Tableau 1).

\subsubsection{Résultats du Test D'Inclinaison}

Le test d'inclinaison était négatif chez tous les patients en décubitus dorsal. En position inclinée le test était positif chez 10 patients (17\%). Après sensibilisation à la Trinitrine, par voie sublinguale, en position inclinée chez 35 patients le test était positif (58\%). Au total le test était positif chez 45 patients (75\%).

Les réponses observées étaient une réponse vasodépressive chez 22 patients, une réponse mixte chez 8 patients, et une réponse cardio-inhibitrice chez 15 patients. Une syncope psychogénique est survenue chez 5 patients.

\subsubsection{Prise en Charge des Patients à Test $D^{\prime}$ 'Inclinaison Positif}

Les patients chez qui le test d'inclinaison avait révélé une syncope vasovagale ont bénéficié des mesures indiquées par les recommandations de l'European Society of Cardiology [6], notamment l'éducation sur la reconnaissance des symptômes précurseurs, d'éviter les facteurs favorisants en privilégiant le décubitus ou à défaut la position assise en cas de reconnaissance des symptômes. Il a été recommandé d’alléger le traitement antihypertenseur chez les patients, en privilégiant l'arrêt ou la diminution de la posologie du traitement diurétique. Les patients ayant eu un test d'inclinaison négatif ont été référés à leur cardiologue traitant.

\subsection{Suivi}

La durée moyenne du suivi dans cette étude était de 6 mois. Au cours du suivi, 5 patients sur 45 (11\%) ont présenté au moins une récidive de syncope. Ainsi, 89\% des patients n’ont pas présenté une récidive des symptômes. Le nombre moyen de syncopes a été réduit. Il a été noté que 03 patients avec test d'inclinaison positif ( $6 \%$ des positifs suivis) et 01 patient avec test d'inclinaison négatif ( $2 \%$ des négatifs suivis) ont eu une récidive d'au moins une syncope ou pré-syncope au cours du suivi. A noter que aucun patient n’a bénéficié de la pose d'un holter implantable ni d'un pace maker. Au cours du suivi seulement 3 patients relativement âgés (moyenne d'âge à 65 ans) avaient une réponse cardio-inhibitrice. Un seul a bénéficié d'implantation d'un pacemaker double chambre; les deux autres sont suivis sans pace maker par manque de moyen financier.

\section{Discussion}

\subsection{Apport Diagnostique du Test D'Inclinaison}

Le test d'inclinaison a été reconnu comme moyen diagnostique de la syncope vasovagale dans notre étude. La durée de la réalisation de cet examen était la principale critique. La provocation médicamenteuse a permis d'augmenter la sensibilité de ce test [7] [8]. Un test d'inclinaison de réalisation plus courte (durée de réalisation de 45 minutes: 10 minutes en décubitus dorsal, 20 minutes phase passive et 15 minutes de provocation) n’a pas montré de différence significative en termes de valeur diagnostique avec la stratégie conventionnelle [9]. Malgré ces données, la stratégie conventionnelle consistant en une phase passive de 30 minutes et une phase de provocation de 20 minutes est toujours recommandée par l'European Society of Cardiology comme stratégie de référence. Le test d’inclinaison reste l'examen de référence dans le diagnostic de la syncope vasovagale. Dans

Tableau 1. Relatif aux caractéristiques cliniques et paraclinique de la population de l’étude.

\begin{tabular}{llr}
\hline \multicolumn{1}{c}{ Caractéristiques cliniques } & \multicolumn{2}{c}{ Effectifs ou moyenne } \\
\hline HTA & $19 / 60$ \\
DiabèteECG sans anomalies significativesFENombre d'épisodes syncopaux & $13 / 60$ & $60 \pm 33 \pm 2$ \\
Durée des symptômes (mois) & $56 / 60$ & 6 mois \\
Durée de suivi (mois) & $6 \pm 3$ & \\
\hline
\end{tabular}

F: sexe féminin; M: sexe masculin; N: nombre; FE: fraction d’éjection systolique du ventricule gauche. La prévalence de l’hypertension artérielle était de $32 \%$ et $22 \%$ pour le diabète. $93 \%$ des patients n’avaient d'anomalie à l'électrocardiogramme; La fraction d'éjection moyenne était $60 \%$ à l'échocardiographie; Sur le plan clinique le nombre moyen d'épisodes syncopaux était de 6. 
notre travail, le test d'inclinaison a, ainsi, permis d’établir le diagnostic positif de syncope vasovagale chez 45 patients soit 75\%, ce qui est déjà un excellent résultat, car on parle ici de patients ayant des syncopes inexpliquées et pour lesquels le test d’inclinaison permet d'établir un diagnostic.

\subsection{Apport Thérapeutique du Test D’Inclinaison}

La reproductibilité des résultats du test d’inclinaison est un autre écueil de ce test diagnostique. En effet, des études d'observation ont montré que la répétition du test d'inclinaison s'associe à une augmentation de taux de réponses négatives [10]. L’hypothèse d’un “apport thérapeutique” du test d’inclinaison a été évoquée. En effet, la survenue d'un épisode vasovagal pendant le test d'inclinaison constitue une sorte de pré-conditionnement et peut empêcher ainsi de nouvelles syncopes. En outre, le test d’inclinaison est généralement effectué sur des patients présentant des épisodes récurrents qui restent inexpliquésmalgré plusieurs examens complémentaires. Ainsi, l'inquiétude sur leur pronostic peut conduire à un certain degré d'anxiété ce qui peut faciliter les récurrences des symptômes. Par conséquent, le caractère bénin de ces syncopes, la reconnaissance de l'étiologie et l'assurance sur le bon pronostic de leurs symptômes peut aider à réduire l'anxiété et donc avoir un impact favorable sur la récidive [11] [12].

Ces explications montrent la diminution significative de la récurrence des symptômes après la réalisation du test d'inclinaison.

Sur 45 patients ayant un test d'inclinaison positif seulement 5 ont fait une récidive de syncope. Ces constats sont identiques à la majorité des études analytiques faites sur le test d’inclinaison et ce indépendamment de la prise en charge thérapeutique [6] [13] [14].

\section{Conclusion}

Le test d'inclinaison a une valeur diagnostique et pronostique dans l'exploration des syncopes inexpliquées probablement d'origine vasovagale. A l'Institut de cardiologie d'Abidjan l'avènement de cet examen a permis de faire le diagnostique des syncopes d’origine vasovagale traitées par excès occasionnant des dépenses supplémentaires.

\section{References}

[1] Blanc, J.J., L’Her, C., Touiza, A., et al. (2002) Prospective Evaluation and Out-Come of Patients Admitted for Syncope over a 1 Year Period. European Heart Journal, 23, 815-820. http://dx.doi.org/10.1053/euhj.2001.2975

[2] Disertori, M., Brignole, M., Menozzi, C., et al. (2003) Management of Patients with Syncope Referred Urgently to General Hospitals. Europace, 5, 283-291. http://dx.doi.org/10.1016/S1099-5129(03)00049-7

[3] Sheldon, R., Rose, S., Flanagan, P., Koshman, M.L. and Killiam, S. (1996) Risk Factors for Syncope Recurrence after a Positive Tilt-Test in Patients with Syncope. Circulation, 93, 973-981. http://dx.doi.org/10.1161/01.CIR.93.5.973

[4] Njoo, Z., Alizadeh, A., Alasti, M., Fadaie, A.A., Haghjoo, M., Fazelifar, A.F., et al. (2007) Correlation between Results of Head-Up Tilt Test and Clinical Features in Patients with Syncope or Presyncope. Journal of Electrocardiology, 40, 200-202. http://dx.doi.org/10.1016/j.jelectrocard.2006.07.006

[5] Kouakam, C. (2011) Test d’inclinaison Modalités, techniques et résultats. Elservier Masson SAS, EMC.

[6] Moya, A., Sutton, R., Ammirati, F., Blanc, J.J., Brignole, M., Dahm, J.B., et al. (2009) Guideline for the Diagnosis and Management of Syncope (Version 2009): The Task Force for the Diagnosis and Management of Syncope of the European Society of Cardiogy (ESC). European Heart Journal, 30, 2631-2671. http://dx.doi.org/10.1093/eurheartj/ehp298

[7] Linzer, M., Yang, E.H., Estes III, N.A., et al. (1997) Diagnosing Syncope. Part II: Unexplained Syncope. Clinical Efficacy Assessment Project of the American College of Physicians. Annals of Internal Medicine, 127, 1352-1355. http://dx.doi.org/10.7326/0003-4819-127-1-199707010-00014

[8] Macebo, P.G., Asirvatham, S.J., Maia, L., Ferreira Neto, E., Zanatta, A., Sobral Neto, J., et al. (2012) Comparison of a Shortened Isosorbide Dinitrate-Potentiated Head-Up Tilt Test Testing with the Conventional Protocol: Tolerance and Diagnostic Accuracy. Pacing and Clinical Electrophysiology, 35, 1005-1011. http://dx.doi.org/10.1111/j.1540-8159.2012.03440.x

[9] Khan, H.F., Hameed, M.A. and Khan, U.A. (2010) Short Duration Head-Up Tilt Test: A Comparison with Conventional Long Protocol in Patients of Orthostatic Ontolerance. Annals of Noninvasive Electrocardiology, 15, 101-106.

[10] Exposito, V., Guzman, J.C., Orava, M., Armaganijan, L. and Morillo, C.A. (2013) Usefulness of the Calgary Syncope Symptom Score for the Diagnosis of Vasovagal Syncope in the Elderly. Placing Clinical Electrophysiol, 36, 37-41. 
http://dx.doi.org/10.1093/europace/eut042

[11] Moya, A., Permanyer-Miralda, G., Sagrista-Sauleda, J., et al. (1955) Limitations of Head-Up Tilt Test for Evaluating the Efficacity of Therapeutic Interventions in Patients with Vaso-Vagal Syncope; Results of a Controlled Study of Etilefrine versus Placebo. Journal of the American College of Cardiology, 25, 65-69. http://dx.doi.org/10.1016/0735-1097(94)00336-O

[12] Petersen, R. (1995) The Clinical Spectrum of Neurocardiogenic Syncope. Journal of Cardiovascular Electrophysiology, 6, 54-58.

[13] Kenny, R.A., Ingram, A., Bayliss, J. and Sutton, R. (1986) Head-Up Tilt: A Useful Test for Investigating Unexplained Syncope. Lancet, 1, 1352-1355. http://dx.doi.org/10.1016/S0140-6736(86)91665-X

[14] Domenichini, G., Diemberger, I., Biffi, M., Martignani, C., Valzania, C., Bertini, M., et al. (2010) Long-Term FollowUp of Patients with Syncope Evaluated by Head-Up Tilt Test. Annals of Noninvasive Electrocardiology, 15, 101-106. http://dx.doi.org/10.1111/j.1542-474X.2010.00349.x 